\title{
Orientation mapping of cp-Ti by reflected polarized light microscopy
}

\author{
Lucia Morales-Rivas $^{1 *}$, Luisa Böhme ${ }^{1}$, Eberhard Kerscher ${ }^{1}$ \\ ${ }^{1}$ Technische Universität Kaiserslautern (TUK), Materials Testing, Gottlieb-Daimler-Str., 67663, Kaiserslautern, Germany \\ *rivas@mv.uni-kl.de
}

\begin{abstract}
$\underline{\text { Abstract }}$
Deformation and fracture mechanisms, and therefore mechanical behavior, in metals and metallic alloys with hexagonal close packed structures, such as $\alpha$-Ti, strongly depend on the crystal c-axis orientation of the grains. Taking advantage of the intrinsic optical anisotropy that these structures present, a computer-aided method based on conventional reflected polarized light microscopy has been developed for c-axis orientation mapping. The application of the method on texture-free and textured commercially pure $\mathrm{Ti}$ is proposed for the examination of relevant crystallographic features.
\end{abstract}

\section{Introduction}

Crystal orientation mapping has become a popular method for the analysis of polycrystalline structures, thanks, to a great extent, to the increasingly higher accessibility to automatic diffraction techniques attached to electron microscopy in scanning mode: electron backscatter diffraction (EBSD) and transmission Kikuchi diffraction, in scanning electron microscopy; and automated crystal orientation mapping, in transmission electron microscopy. They are not free of limitations though: the high spatial resolution is achieved at the expense of reduced sample dimensions and scanned area. Additionally, the sample preparation is highly demanding for both transmission and backscatter conditions.

If the microstructure to be analyzed does not require these high resolution techniques, light microscopy can still be an alternative to explore. When dealing with birefringent structures, such as hcp, which is optically anisotropic, polarized light microscopy (PLM) produces brightness contrast according to the c-axis orientation of the examined grains. In the case at hand, $\alpha$-Ti, non-scanning plane-polarized light microscopy provides with a rapid overview of the microstructure and is still able to partly reveal local crystallographic information. When assisted by image processing tools, a semi-automated PLM c-axis orientation mapping is feasible [1], as will be later explained. The c-axis orientation in each grain is determined with respect to the light beam direction and to the light vibration plane, which can be "translated" into the sample reference frame (global coordinates). Relative c-axis orientations, i.e., c-axis misalignments between different crystals are therefore also accessible.

In the following, the influence of the c-axis orientation on the deformation mechanisms of $\alpha$-Ti will be rationalized. It will help contextualize the scope of the subsequently briefly described semi-automated PLM method for the c-axis orientation mapping. Examples of PLM and EBSD maps will be shown for comparison.

\section{On the c-axis orientation ruling deformation mechanisms}

Hexagonal crystal structures have a limited number of independent slip systems at room temperature conditions, compromising the plastic deformation compatibility of polycrystalline structures. Twinning is the complementary deformation mechanism which, in these structures and under some conditions, guarantees certain material ductility. The extension to which the crystal c-axis orientation correlates with these two competing and complementary mechanisms, slip and twinning, will be elucidated below.

Regarding the determination of the activated slip systems in $\alpha$-Ti and its alloys, the ratio between the critical resolved shear stress of the two main slip systems,

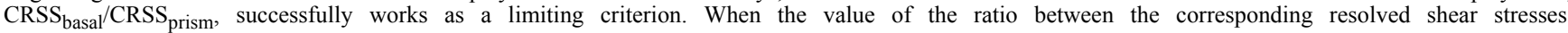
$\mathrm{RSS}_{\text {basal }} / \mathrm{RSS}_{\text {prism }}$, is above it, basal slip is favored; whereas for $\mathrm{RSS}_{\text {basal }} / \mathrm{RSS}_{\text {prism }}<\mathrm{CRSS}_{\text {basal }} / \mathrm{CRSS}_{\text {prism }}$, prismatic slip is favored instead [2]. For the case of pure Ti, the ratio $\mathrm{CRSS}_{\text {basal }} / \mathrm{CRSS}_{\text {prism }}$ is high [2]. This fact, together with the applicability of a global Schmid factor (that is, the determination of RSS from global stresses), explains the following frequent observation: grains with their c-axis perpendicular to tensile stress direction are "soft" (yield easily), because of the favored slip on one or more of the three prismatic slip systems; whereas those with their c-axis parallel to tensile stress direction are "hard" [3]. For intermediate orientations between these two extreme cases or under more complex stress states, deformation mechanisms, even if considering only slip, are strongly influenced by the misorientations between adjacent grains, as they imply misalignment of the slip systems, leading to a higher or lower deformation compatibility [4]. Beyond the applicability of c-axis orientation mapping, the examination of basal pole figures, i.e., c-axis orientation distribution, is also relevant [5]. Such a global analysis will prove the existence, progressive appearance or absence of textures in $\alpha$-Ti, with strong implications in terms of its strain hardening behavior.

As mentioned, twinning is the other main deformation mechanism in hexagonal structures, favored against slip at low temperatures and high strain rates. Different type of twins can appear each corresponding to a specific misorientation [6]. In Table 1 the expected types of deformation twins for Ti at room temperature and their misorientation with respect to the matrix in the rotation axis/misorientation angle convention are shown. Two kind of twins exist (Table 1): Tensile (or extension) twins and compression (or contraction) twins, according to the strain to which the c-axis of the twin is subjected. It can be noticed that for all type of twins, the rotation axis belongs to the basal plane, i.e., the misorientation between the matrix and the twin always consists in a pure rotation around a crystal axis normal to the c-axis. Therefore, this is a particular case where the misorientation angle and the c-axis misalignment angle between the twin and the matrix are coincident. Since the misorientation angle is unique for each kind of twin, this value is in theory enough to define the twin type, meaning that PLM might be an appropriate technique for the twin type identification.

Table 1. Commonly observed twinning systems in Ti at room temperature (misorientation angles have been rounded to integer values).

\begin{tabular}{||c|c|c|}
\hline Twinning system & Rotation axis & Misorientation angle \\
\hline \hline Extension twin (TT1) & $<1-100>$ & $35^{\circ}$ \\
\hline \hline Extension twin (TT2) & $<11-20>$ & $85^{\circ}$ \\
\hline \hline Contraction twin (CT1) & $<1-100>$ & $65^{\circ}$ \\
\hline
\end{tabular}

The complex nucleation and growth mechanisms in Ti are far from being understood. However, twinning involves microstructural changes that can be classified according to their effect on the stress-strain response [8]. On one hand, strain hardening is believed to come from the Hall-Petch effect, that is, the refinement of the microstructure by the proliferation of grain boundaries; and from the Basinski mechanism, which implies the conversion of glissile dislocations in the matrix into sessile dislocations when they get inherited by the twin. These two phenomena are consequence of the orientation relationship between matrix and twin, and in the case of Hall-Petch, also of the twin boundary nature, which get defined by the type of twinning. On the other hand, an either hardening or softening effect which can appear as the texture evolves owing to twin variant selection. Within the scope of the present work, for the former case (Hall-Petch and Basinski effects), the misorientation between matrix and twin, which determines its contribution to the strain hardening, can be completely defined by their c-axis misalignment (relative 
c-axis orientation). For the latter case (twinning texture), the hardening or softening behavior is attributed to the evolution of crystal orientation (with respect to global coordinates, absolute crystal orientation), for which the absolute c-axis orientation is a relevant parameter to be obtained, as previously mentioned.

Summarizing, from the point of view of deformation mechanisms, and thus also fracture mechanisms, in $\alpha$-Ti and its alloys both mapping and distribution determination of c-axis orientation, and twin identification, are of high interest. Additionally, such a characterization is also key for the understanding of in-situ properties dependent on the energy of the crystal plane at the surface of the component [9] or a fatigue crack propagation path [10].

\section{Description of c-axis orientation mapping based on polarized light microscopy and examples}

As mentioned, PLM applied on a polished metallic sample with an optically uniaxial crystal structure is partially sensitive to crystal orientation, i.e., grains can be revealed by brightness contrast. Figure 1 a shows a PLM picture of deformed commercially-pure (cp) Ti, while Figure 1 . b shows an EBSD IPF map of the same area, for comparison. There is a clear presence of deformation twins (both extension and contraction twins), which are morphologically better revealed in the PLM picture, due to a lower sensitivity of PLM to the surface roughness.

Particularly, working with cross polarizer position, the brightness produced by the interaction of plane-polarized light with the polished surface of the sample is a function of [1]:

-The inclination: angle between the c-axis of each observed crystal and the propagation direction of the incident light (normal to the sample surface).

-The azimuth: angle between the projection of the crystal c-axis on the sample surface and the plane of vibration of the incident light (normal to the transversal direction, TD).
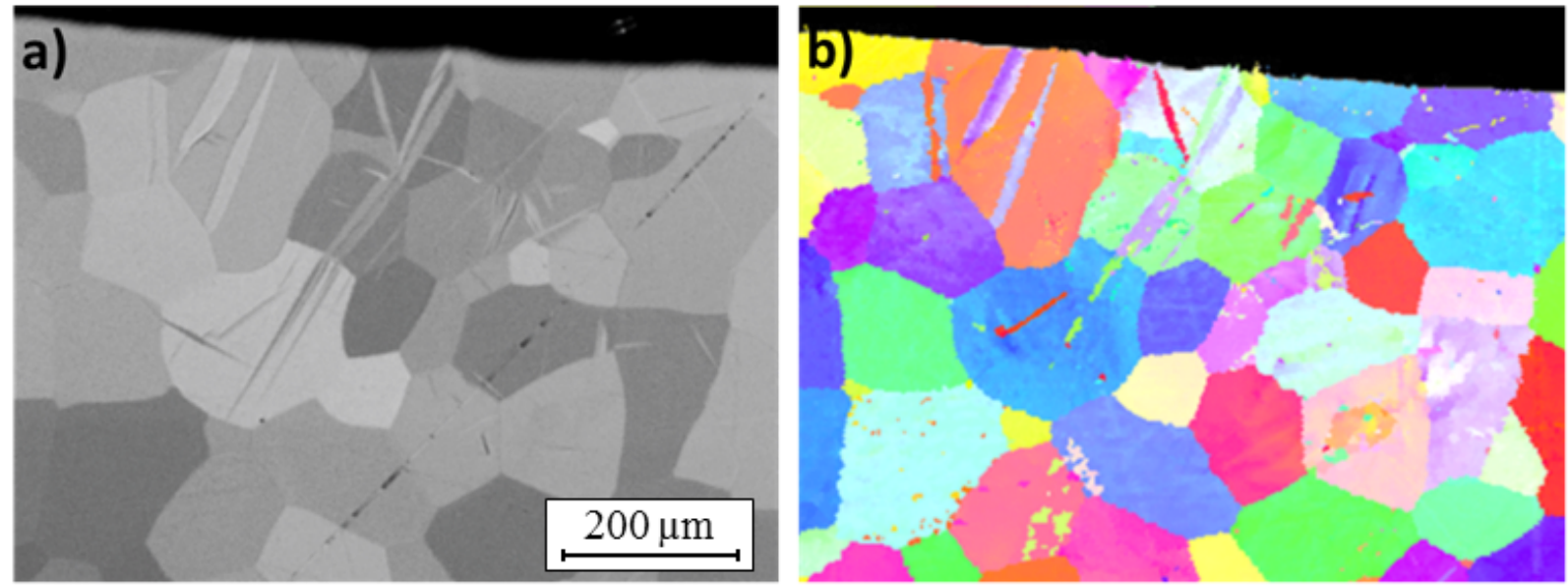

Figure 1. PLM picture (a) and cleaned EBSD ipf map (b) of the same area of deformed cp-Ti. The observed surface corresponds to the lateral side of a beam subjected to a three-point bending test, and the area is close to the zone of maximum deflection at the compression side.

In order to reduce this uncertainty and therefore to discern inclination and azimuth, different relative orientations between the crystal and the light coordinates must be evaluated. In reflected light microscopy the sample surface must remain always perpendicular to the propagation direction of the incident light, implying that the inclination cannot be varied. On the contrary, for the mentioned purpose, the azimuth can be varied by rotation around an axis parallel to the propagation direction of the incident light, and the reflected light intensity (brightness) evolution tracked. The brightness of each crystal evolves with the angle according to a sinusoidal function with a periodicity of $180^{\circ}$ (Figure 2): the phase shift is directly proportional to the azimuth, while the sine amplitude (half the theoretical difference between the maximum a minimum reflected intensity values) is function of the inclination.
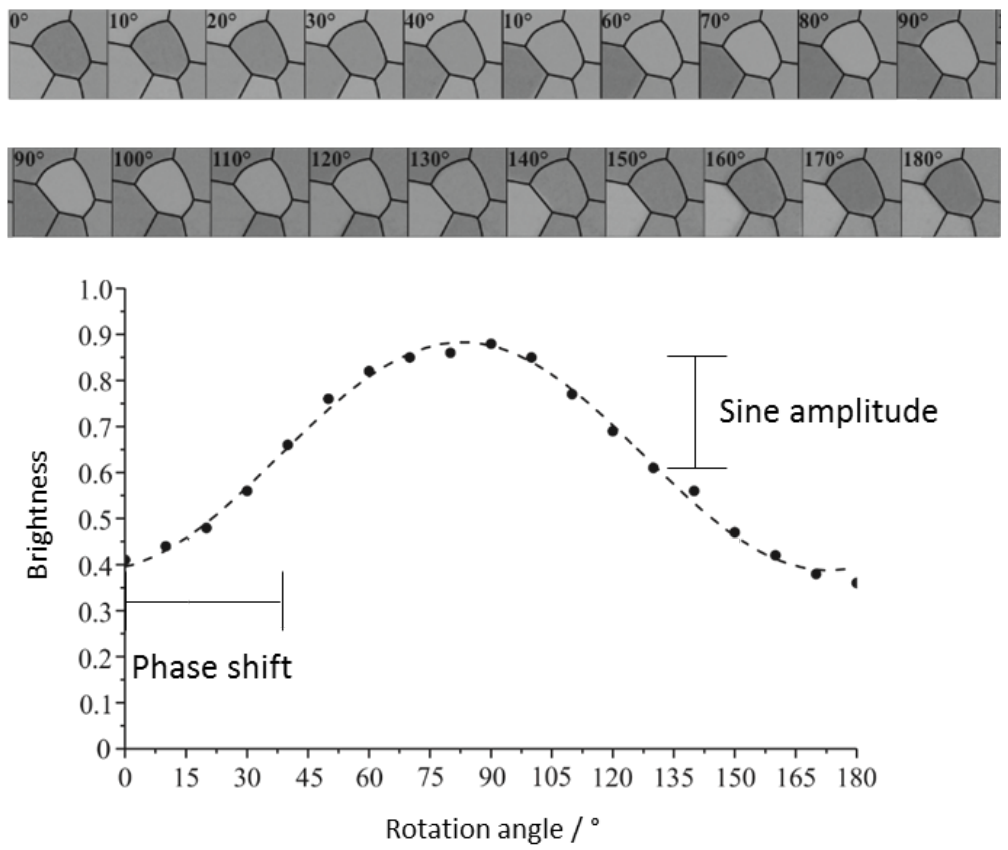

Figure 2. Example of brightness evolution of a grain of $\alpha$-Ti, as observed by using PLM, as the sample is rotated on the stage. (Modified figure from ref. [1]). 
A recent method has been developed by the authors [1], a semi-automated approach for the previously explained analysis, involving PLM-based experimental procedure, computing-assisted processing of the pictures, and, under certain circumstances, calibration by means of EBSD. First, the sample on the stage of the microscope is rotated by steps of a certain number of degrees, acquiring a PLM picture every time. After an appropriate virtual rotation of the PLM pictures back to the original position, grain boundaries are detected in each one based on gray contrast. The grain boundaries are used for an automated superimposition of the pictures and the subsequent grain recognition [11]. Subsequently, the gray evolution for each grain is fitted to a sine function, from which the phase shift and the amplitude values are obtained, as explained. The conversion of the phase shift into the azimuth angle is self-consisting for each grain, regardless of the absolute values of the brightness. However, it does not hold true for the conversion of the sine amplitude into the inclination angle. In this sense, different scenarios must be considered. In cases in which the microstructure presents a strong texture or the PLM picture area is too small to be representative of the whole microstructure, a calibration pre-step is compulsory. It can be done by applying both the PLM method and an alternative reliable technique such as EBSD to the same area of the sample surface. For the same material, same surface conditions, and same settings of the microscope, a calibration curve for the conversion between the PLM-based sine amplitude into the EBSD-based inclination angle is calculated [1]. Otherwise, if the orientations of the grains contained within the PLM picture are randomly spread filling the whole Euler space, i.e., there is not texture, the calibration can be skipped. In such a case, the sine amplitude - inclination conversion curve can be determined, instead, by assigning an inclination of $90^{\circ}$ to the grain presenting the maximum recorded sine amplitude, and an inclination of $0^{\circ}$ to the grain with the minimum sine amplitude.

For orientation mapping, EBSD-like datasets are generated from the azimuth and inclination values determined by the PLM method. In Figure 3, details of crystal direction maps from EBSD and from the PLM method are shown, for the same area of a texture-free cp-Ti sample. The correlation of the EBSD and PLM results is in most cases qualitatively successful.

EBSD

a)

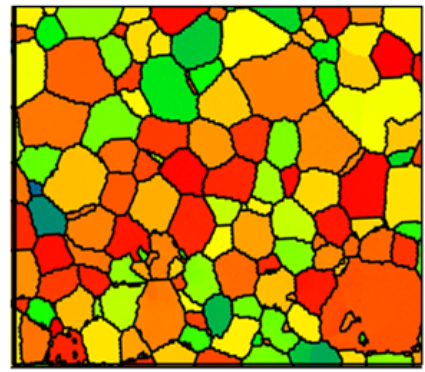

$1 \mathrm{~mm}$

c)

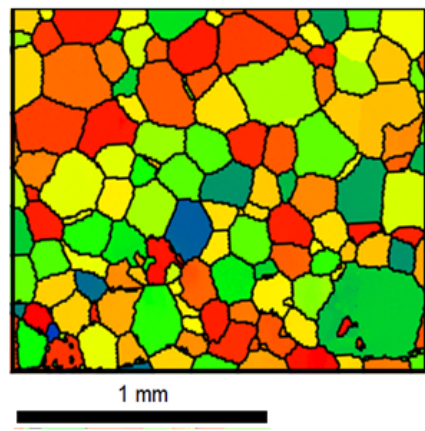

b)

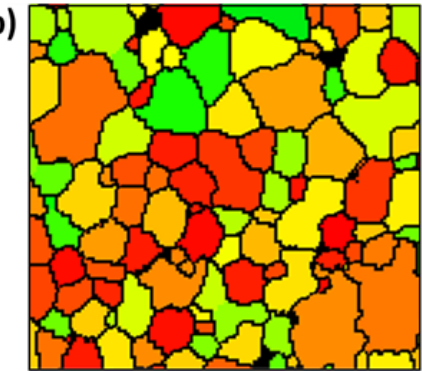

$1 \mathrm{~mm}$

d)

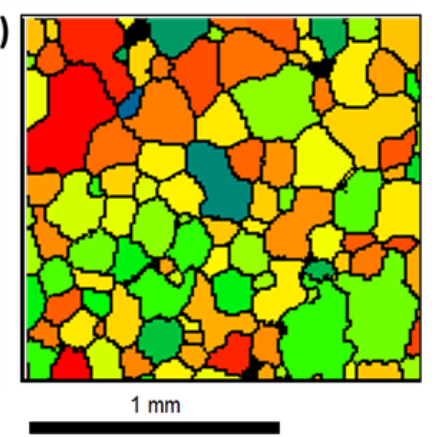

PLM

Figure. 3. $<0$ o $01>$ crystal direction maps of texture-free $\alpha$-Ti: color-coded according to the angle between the c-axis of the grains and TD from EBSD (a) and from the PLM method (b); and color-coded according to the angle between the crystal c-axis of the grains and the normal to the surface from EBSD (c) and from the PLM method (d). Grain boundaries (for a misorientation of $10^{\circ}$ ) and unindexed points are in black. (Modified figure from ref. [1]).

The method has been also successfully applied to textured samples, from which basal pole figures can be regularly calculated. As observed in Figure 5, anisotropy in the caxis orientation distribution is correctly revealed by the PLM method.
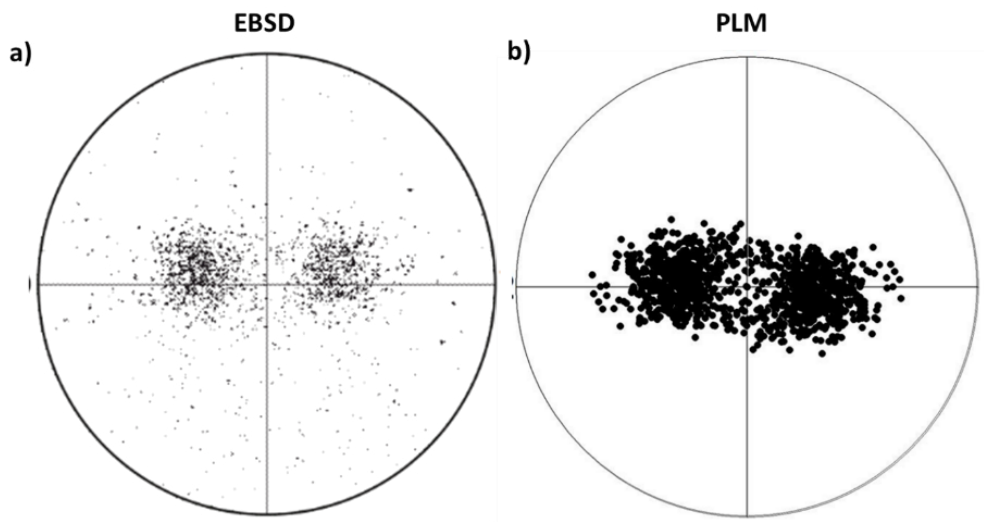

Figure 4. $<000$ 1 $>$ pole figures of textured $\alpha$-Ti from EBSD (a) and from the PLM method (b). The plane of sight is the sample surface, normal to the rolling direction. (Modified figure from ref. [1]).

\section{Conclusion}

A new semi-automated method for crystallographic characterization of hep metal based on reflected PLM has been presented. The method is suitable for c-axis orientation mapping and distribution determination, for both texture-free and textured cp-Ti, and has an interesting potential for the twin type determination, all of them relevant crystallographic features in terms of mechanical behavior. 


\section{Acknowledgements}

This research was funded by the German Research Foundation (DFG) within the Collaborative Research Center 926 "Microscale Morphology of Component Surfaces" project number 172116086 .

\section{$\underline{\text { References }}$}

1. Böhme, L.; Morales-Rivas, L.; Diederichs, S.; Kerscher, E. Mater. Charact. 2018, 145, 573-581.

2. Wang, L.; Zheng, Z.; Phukan, H.; Kenesei, P.; Park, J.S.; Lind, J.; Suter, R.M.; Bieler, T.R. Acta Mater. 2017, 132, 598-610.

3. Dunne, F.P.E.; Walker, A.; Rugg, D. Proceedings of the Royal Society A: Mathematical, Physical and Engineering Science 2007, 463, 1467-1489.

4. Livingston, J.D.; Chalmers, B. Acta Metall. 1957, 5, 322-327.

5. Zarkades, A.; Larson, F.R. A review of textures found in commercial titanium sheets. AMMRC TR 71-60, AD 745123; Army materials and mechanics research center: Watertown, Massachusetts 02172, 1971.

6. Lainé, S.J.; Knowles, K.M. Philos. Mag. 2015, 95, 2153-2166.

7. Bozzolo, N.; Chan, L.; Rollett, A.D. J. Appl. Crystallogr. 2010, 43, 596-602.

8. $\quad$ Salem, A.A.; Kalidindi, S.R.; Doherty, R.D.; Semiatin, S.L. Metall. Mater. Trans. A 2006, 37, 259-268.

9. Majid, H.; Philippe, B.; Arash, S.; Fereshteh, A.; A., S.J.; Hojatollah, V. Journal of Biomedical Materials Research Part A 2014, 102, 3631-3638.

10. Peters, M.; Gysler, A.; LÜtjering, G. Metall. Mater. Trans. A 1984, 15, 1597-1605.

11. Morales-Rivas, L.; Diederichs, S.; Böhme, L.; Gordo, E.; Hebestreit, A.; Kerscher, E. Practical Metallography 2018, 55, 678692. 